\title{
Vibration Research on Biomechanics Effects of Different Frequencies in Gastrocnemius in Rats
}

\author{
Fuming Gu \\ Department of Sports Medicine, Chengdu Sport University, Chengdu, China
}

Keywords: Biomechanics; structural mechanics; whole-body vibration; frequency

\begin{abstract}
The aim of this study was to explore the biomechanical effects of different frequencies whole-body vibration training on gastrocnemius in rats. The conclusion showed that (a) there was significant difference between high frequency group and control group, and there was extremely significant difference between low frequency group and control group in characteristics of maximal load. There was extremely significant difference between low frequency group and control group, low frequency group and middle frequency in characteristics of energy absorbed at the maximal load, and (b) there was significant difference between low frequency group and control group, extremely significant difference between low frequency group and control group in characteristics of modulus of elasticity; there was extremely significant difference between low frequency group and control group, low frequency group and middle frequency, significant difference between high frequency and low frequency group and high frequency group in characteristics of maximal stress.
\end{abstract}

\section{Introduction}

In the literature of vibration training, there many researchers have performed some researches on the effects of vibration. For instance, Chen et al. (Chen et al., 2014) investigated whether lowmagnitude whole-body vibration could enhance the protective effect of ALE on bone properties in ovariectomized rats. The conclusions revealed that WBV enhanced the effect of alendronate in ovariectomized rats by inducing further improvements in trabecular architecture. Łochyński et al. (2013) determine the effects of 5-week whole-body vibration on contractile parameters and forcefrequency relationship of functionally isolated motor units of the rat medial gastrocnemius muscle. Nowak et al. (2012) examined whether short-lasting whole-body vibration (WBV) was sufficient to induce bone metabolic and immunological response, and whether the increasing volume of vibration training modifies changes in bone and modulate immunological indices. Maddalozzo et al. (2008) evaluated the effects of whole-body vibration on fat, bone, leptin and muscle mass in rats. Felicitas Wirth et al. (2013) provided the first evidence for functional benefits of WBV in an animal Spinal Cord Injured rat model and warrant further preclinical investigations to determine mechanisms underpinning this noninvasive, inexpensive, and easily delivered potential rehabilitation therapy for Spinal Cord Injured rat. Pasqualini et al. (2013) investigated the bone effects of different vibration frequencies at constant g level, and vertical Whole-body vibration was delivered at $0.7 \mathrm{~g}$ and 8,52 or $90 \mathrm{~Hz}$ sinusoidal vibration to mature male rats $10 \mathrm{~min}$ daily for 5days/week for 4weeks.

In this study, the author explored the effects of vibration on the biomechanical effects of different frequencies whole-body vibration training on gastrocnemius in rats.

\section{Materials and Methods}

\section{Experimental Animals and Feeding Methods}

At the Experimental Animal Center under School of West China Medicine, Sichuan University, 60 healthy male SD rats with their weights ranging from $180 \mathrm{~g}$ to $210 \mathrm{~g}$ are nurtured and raised for 3 months in rack cages, with 5 rats per cage. The temperature for the movement and feeding environments is set between $18^{\circ} \mathrm{C}$ and $24^{\circ} \mathrm{C}$, and the relative humidity ranges from $45 \%$ to $55 \%$. They are exposed to natural light. In addition, all fed with standard pellet feed for rodents, and 
freely permitted to intake food and water.

\section{Scheme for Grouping and Training Animals}

After one week's adaptive feeding, the rats are randomly divided into the following four groups: the rest control group $(\mathrm{C})$, the $25 \mathrm{~Hz}$ vibration training group $(\mathrm{L})$, the $35 \mathrm{~Hz}$ vibration training group $(\mathrm{M})$, and the $45-\mathrm{Hz}$ vibration training group $(\mathrm{H})$, with 15 rats per group. Vibration training is provided to each training group of rats on the Power Plate vibration training platform. When being trained, 15 rats of the same group are simultaneously placed on the vibrating platform. Each rat is respectively deployed to self-made organic glassware where such rat is restrained from standing up, and such rat's lower limbs are subject to vibrating stimulation under gravity-resistant conditions. Each day requires 2 vibration training sessions with each training session spanning 15 min. The interval between the two vibration training sessions is $5 \mathrm{~min}$. The vertical amplitude of each group is $3 \mathrm{~mm}$. Such training is required 5 days each week with 2 days for rest, and lasts for 8 weeks.

\section{Animal Sampling}

Sampling is conducted within $24 \mathrm{~h}$ following the final vibration training for each group of rats. The $10 \%$ chloral hydrate solution $(3 \mathrm{~mL} / \mathrm{kg}$ ) is used to anesthetize such rats via intraperitoneal injection. The rats' right gastrocnemius muscles are sampled. Then, the extra peripheral tissues are cleaned away while leaving the tendons on both sides. The sample is wrapped in gauze, which has been soaked in a saline solution, and is immediately placed in a cryogenic vial precooled with liquid nitrogen. After that, the vial is stored at $-80^{\circ} \mathrm{C}$ in a refrigerator for further testing.

\section{Mechanical Properties Testing}

At the Key Provincial Biomechanics Laboratory of Sichuan University, the thawed gastrocnemius muscles for testing are sandwiched in a test fixture and then placed on an electronic universal testing machine (AGIS-MS model) developed by Shimadzu. In a constant-temperature and constant-humid laboratory, a biomechanics test is run on such gastrocnemius muscles. Before official testing, a preliminary calibration process for testing items is run. The preliminary calibration is run 10 times for cyclic tensile loading. The load ranges from 0 to $3 \mathrm{~N}$, and the loading rate is $3 \mathrm{~mm}$ per min. After the preliminary calibration test, an official tensile failure test with a loading rate of $3 \mathrm{~mm}$ per min is run. The successful experimental standard is that the tested items do not break off from their test fixture. Meanwhile, the load deformation curve is recorded. During the testing period, the needle fully injected with a saline solution is constantly dripped while maintaining its moisture. Thereafter, the TRAPEZIUM 2 software on the electronic universal testing machine processes the data and outputs structural and mechanical parameters (including the maximal load and the energy absorbed at the maximal load) and mechanical parameters of materials (including modulus of elasticity, maximal stress,).

\section{Data analysis}

All data collected from this study were analyzed with SPSS 16.0 for Windows. One way ANOVA, LSD multiple comparison were used in this study. All significant level was set at $\mathrm{P}<0.05$ level, and extremely significant level at $\mathrm{p}<0.01$.

\section{Results and analysis}

\section{Characteristics of structural mechanics}

The one way ANVOA test of the structural mechanics parameters with LSD multiple comparison indicated the results as follows: (a) in the maximal load ANVOA test, $\mathrm{F}=3.245, \mathrm{P}=$ $0.031<0.05$, it showed that there are significant difference between groups. Further LSD multiple comparison showed (see figure 1) that there was significant difference between high frequency group and control group $(\mathrm{p}=0.019<0.05)$, there was extremely significant difference between low frequency group and control group $\mathrm{p}=0.009<0.01$ ). There was no significant difference between other groups, and (b) in the energy absorbed at the maximal load ANVOA test, $\mathrm{F}=5.301, \mathrm{P}=$ $0.003<0.01$, it showed that there are extremely significant difference between groups. Further LSD multiple comparison showed (see figure 2) that there was extremely significant difference between low frequency group and control group $(\mathrm{p}=0.001<0.05)$, low frequency group and middle frequency $(p=0.005<0.01)$. There was no significant difference between other groups. 


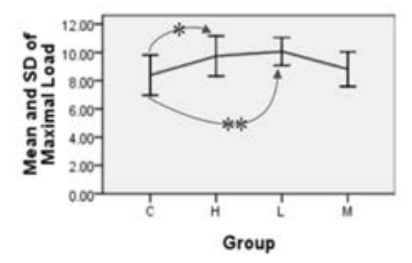

Figure 1. Comparison of characteristics of maximal stress between different vibration frequencies Note: * indicates significant difference at $\mathrm{P}<0.05$, ** indicates extremely significant difference at $\mathrm{P}<0.01$

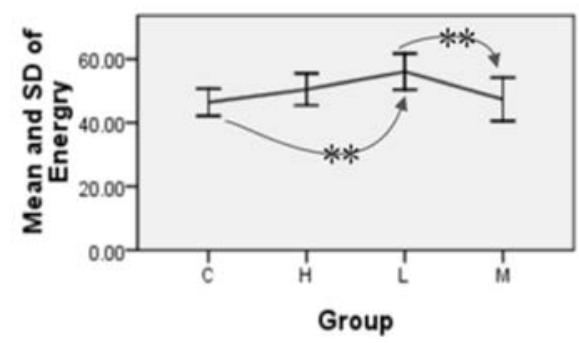

Figure 2. Comparison of characteristics of energy absorption between different vibration frequencies

Note: * indicates significant difference at $\mathrm{P}<0.05$, ** indicates extremely significant difference at

\section{Characteristics of mechanics of material}

The one way ANVOA test of the mechanics of material parameters with LSD multiple comparison indicated the results as follows: (a) in the modulus of elasticity ANVOA test, $\mathrm{F}=3.805$, $\mathrm{P}=0.017<0.05$, it showed that there are significant difference between groups. Further LSD multiple comparison showed (see figure 3) that there was significant difference between low frequency group and control group $(\mathrm{p}=0.040<0.05)$, there was extremely significant difference between low frequency group and control group $(\mathrm{p}=0.002<0.01)$. There was no significant difference between other groups, and (b) in the maximal stress ANVOA test, F=7.974, $\mathrm{P}=$ $0.000<0.01$, it showed that there are extremely significant difference between groups. Further LSD multiple comparison showed (see figure 4) that there was extremely significant difference between low frequency group and control group $(\mathrm{p}=0.000<0.01)$, low frequency group and middle frequency. There was significant difference between high frequency and low frequency group and high frequency group $(\mathrm{p}=0.044<0.05)$. There was no significant difference between other groups.

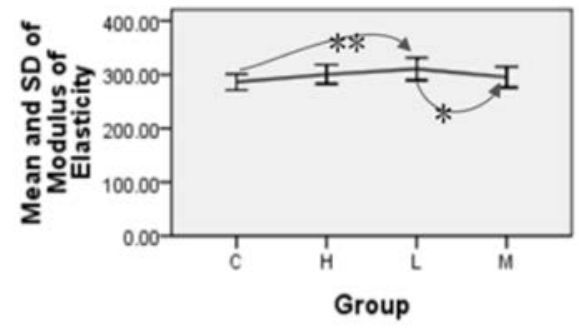

Figure 3. Comparison of modulus elasticity between different vibration frequencies

Note: * indicates significant difference at $\mathrm{p}<\underset{\mathrm{P}<0.01}{0.05}$, indicates extremely significant difference at

The effect of vibration training is affected by amplitude, frequency, direction, duration, etc. of vibration waves. In a report about the effect of vibration frequency on athletic ability, it is shown in a research by Liu Beixiang (2011) that the effect of vibration training increases significantly with the increase of frequency in the vibration frequency range of $15 \sim 45 \mathrm{~Hz}$. It is also found in the research about vibration training with the same amplitude but different frequencies on lower limb muscles of athletes by Ren Manying et al. (2008) that different vibration frequencies create different impacts on the effect of vibration. Vibratory stimulation with the same amplitude (4mm) and frequency of $35 \sim 50 \mathrm{~Hz}$ has a better effect on the maximum strength, speed strength, explosive force and endurance of muscles than the vibration stimulation with the frequency of $25 \sim 35 \mathrm{~Hz}$. 


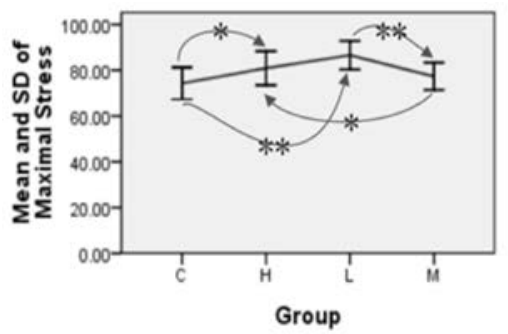

Figure 4. Comparison of maximal stress between different vibration frequencies Note: * indicates significant difference at $\mathrm{p}<0.05, * *$ indicates extremely significant difference at $\mathrm{P}<0.01$

It is indicated in this research that the maximum load, absorption of energy, elastic modulus, maximum stress and other dynamics parameters of gastrocnemius muscle of rats in group $25 \mathrm{~Hz}$ and $45 \mathrm{~Hz}$ are higher than those of rats in the control group, while there is no significant difference in dynamics parameters of gastrocnemius muscle between rats in group $35 \mathrm{~Hz}$ and control group, which shows that vibration training in group $25 \mathrm{~Hz}$ and $45 \mathrm{~Hz}$ can improve the strength of muscles of rats, while the vibration training does not have obvious effect on group $35 \mathrm{~Hz}$. The reason for this may be that frequency of vibration in group $35 \mathrm{~Hz}$ is equal or close to natural frequency of muscles of lower limb of rats. It can be known from strength formula of mechanical waves $I=\rho u A^{2} \omega^{2} / 2$ ( $\rho$ is density of medium, $u$ is wave speed, $A$ is amplitude and $\omega$ is frequency of vibration in the formula): the strength of mechanical waves is proportional to the square of amplitude and frequency. When external vibration frequency applied to rats is equal or close to the natural frequency in the forced vibration system, the vibration amplitude A of the system will increase significantly. In this case, rats will suffer from physical discomfort due to large vibration load. It could be confirmed indirectly from the growth and mental status of each training group: During training, rats of group $35 \mathrm{~Hz}$ have relatively worse mental status with four of them dead, while mental status of rats in other groups are good without death.

Therefore, the frequency for vibration training shall not be from regions around the natural frequency of parts to be trained, and the range of such region is to be further discussed. On this basis, relatively high vibration frequencies (even higher than that of parts to be trained) can be selected for training, so as to generate sufficient stimulus for the muscle and get a fine result from the vibration exercise. Different body parts, however, have significant different natural frequencies and there is no sufficient related literature about the natural frequencies of soft tissues of rats, so a lot of researches shall be made to determine the proper range of vibration frequencies.

From the above test, it can be concluded that the vibration training in low and high frequency can increase the maximal load and energy absorbed at the maximal load, but the middle frequency has no such function. The author assumes that there is a frequency zone in $30 \mathrm{~Hz}$ or so, in this zone, vibration training has no function on subject muscle.

\section{Conclusions}

There was significant difference between high frequency group and control group, and there was extremely significant difference between low frequency group and control group in characteristics of maximal load. There was extremely significant difference between low frequency group and control group, low frequency group and middle frequency in characteristics of energy absorbed at the maximal load.

There was significant difference between low frequency group and control group; there was extremely significant difference between low frequency group and control group in characteristics of modulus of elasticity; there was extremely significant difference between low frequency group and control group, low frequency group and middle frequency. There was significant difference between high frequency and low frequency group and high frequency group in characteristics of 
maximal stress.

\section{Acknowledgements}

This study was supported by National Natural Science Foundation of China (No: 11272068).

\section{References}

[1] Chen, Guo-Xian; Zheng, Shuai; Qin, Shuai; Zhong et al..Effect of Low-Magnitude Whole-Body Vibration Combined with Alendronate in Ovariectomized Rats: A Random Controlled Osteoporosis Prevention Study. PLoS ONE. May2014, Vol. 9 Issue 5, p1-8.

[2] Felicitas Wirth; Greta Schempf; Gregor Stein et al.Whole-Body Vibration Improves Functional Recovery in Spinal Cord Injured Rats.Journal of Neurotrauma. Mar2013, Vol. 30 Issue 6, p453468

[3] Liu Beixiang.2011. Effects of vibration wave direction and frequency on vibration training. Journal of Wuhan Institute of Physical Education.,Vol. 45,Issue 6.p83-87

[4] Łochyński, Dawid; Bączyk, Marcin; Kaczmarek, Dominik; Rędowicz, Maria Jolanta; Celichowski, Jan; Krutki, Piotr, Adaptations in physiological properties of rat motor units following 5 weeks of whole-body vibration. Applied Physiology, Nutrition \& Metabolism Sep2013, Vol. 38 Issue 9, p 913

[5] Maddalozzo, G F; Iwaniec, U T; Turner, R T; Rosen, C J; Widrick, J J. Whole-body vibration slows the acquisition of fat in mature female rats.International Journal of Obesity. Sep2008, Vol. 32 Issue 9, p1348-1354.

[6] Nowak, Alicja; Pawlak, Maciej; Brychcy, Martyna; Celichowski, Jan; Krutki, Piotr,Effects of brief whole-body vibration on bone metabolic and immunological indices in rats.Studies in Physical Culture \& Tourism 2012, Vol. 19 Issue 2, p73 4.

[7] Pasqualini, Marion et al. 2013. Skeletal site-specific effects of whole body vibration in mature rats: From deleterious to beneficial frequency-dependent effects. BONE. Vol. 55 Issue 1, p69-77

[8] Ren Manying, Pan Lei, Wei Yongwang.2008. An Experimental Research of the Effects of Strength Training under Vibration Stimulation with Different Frequency on Knee Muscles. Journal of Capital Institute of Physical Education, Vol 20 (3), p71-75. 